\title{
Multiple steady-states in the terrestrial atmosphere-biosphere system: a result of a discrete vegetation classification?
}

\author{
A. Kleidon ${ }^{1}$, K. Fraedrich ${ }^{2}$, and C. Low $^{3}$ \\ ${ }^{1}$ Biospheric Theory and Modelling Group, Max-Planck-Institut für Biogeochemie, Postfach 1001 24, 07701 Jena, Germany \\ ${ }^{2}$ Meteorologisches Institut, Universität Hamburg, Bundesstr. 55, 20146 Hamburg, Germany \\ ${ }^{3}$ Department of Geography, University of Maryland, 2181 Lefrak Hall, College Park, MD 20742, USA
}

Received: 19 January 2007 - Published in Biogeosciences Discuss.: 22 February 2007

Revised: 24 August 2007 - Accepted: 24 August 2007 - Published: 28 August 2007

\begin{abstract}
Multiple steady states in the atmospherebiosphere system can arise as a consequence of interactions and positive feedbacks. While atmospheric conditions affect vegetation productivity in terms of available light, water, and heat, different levels of vegetation productivity can result in differing energy- and water partitioning at the land surface, thereby leading to different atmospheric conditions. Here we investigate the emergence of multiple steady states in the terrestrial atmosphere-biosphere system and focus on the role of how vegetation is represented in the model: (i) in terms of a few, discrete vegetation classes, or (ii) a continuous representation. We then conduct sensitivity simulations with respect to initial conditions and to the number of discrete vegetation classes in order to investigate the emergence of multiple steady states. We find that multiple steady states occur in our model only if vegetation is represented by a few vegetation classes. With an increased number of classes, the difference between the number of multiple steady states diminishes, and disappears completely in our model when vegetation is represented by 8 classes or more. Despite the convergence of the multiple steady states into a single one, the resulting climate-vegetation state is nevertheless less productive when compared to the emerging state associated with the continuous vegetation parameterization. We conclude from these results that the representation of vegetation in terms of a few, discrete vegetation classes can result (a) in an artificial emergence of multiple steady states and (b) in a underestimation of vegetation productivity. Both of these aspects are important limitations to be considered when global vegetation-atmosphere models are to be applied to topics of global change.
\end{abstract}

Correspondence to: A. Kleidon

(akleidon@bgc-jena.mpg.de)

\section{Introduction}

The primary example for multiple steady states in the climate system is related to the ice-albedo feedback, and was first demonstrated with a simple energy balance model by Budyko (1969) and Sellers (1969). Interactions and, more specifically, associated positive feedbacks are the cause for the emergence of multiple steady states: Surface albedo strongly affects surface temperature, but the prevailing surface temperature affects the extent to which ice and snow is present, thereby affecting surface albedo. For a certain range of solar luminosity values, the positive ice-albedo feedback can then lead to two stable steady states: a cold "Snowball Earth" with abundant ice cover, and a warm, ice-free Earth. As a consequence, the emergent climatic state depends on the initial conditions, and the climate system can react to perturbation by drastic change due to the transition into a different steady state (e.g. Fraedrich, 1979).

When applied to the climate system over land, multiple steady states can emerge from the interactions between terrestrial vegetation and the overlying atmosphere (e.g. Pielke et al., 1993; Claussen, 1994). Vegetation activity strongly modulates the exchange of energy and water at the land surface. The resulting feedbacks can broadly be summarized by two positive biogeophysical feedbacks that operate in regions in which water availability and temperature are the dominant climate-related limitations on vegetation productivity (e.g. Kleidon and Fraedrich, 2005):

1. In regions where temperature limits productivity (through the length of the growing season, primarily in high latitudes), forest masks the effect of snow cover, resulting in a lower surface albedo when snow is present. This leads to an earlier springtime warming, which accelerates snowmelt and extends the growing season (Otterman et al., 1984; Harvey, 1988; Bonan et al., 1992). Overall, the effect of boreal forest on snow albedo is characterized by a positive feedback: the presence of

Published by Copernicus Publications on behalf of the European Geosciences Union. 
(a)

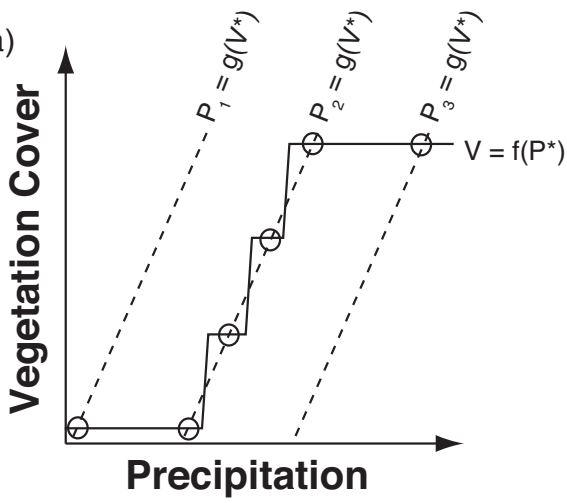

(b)

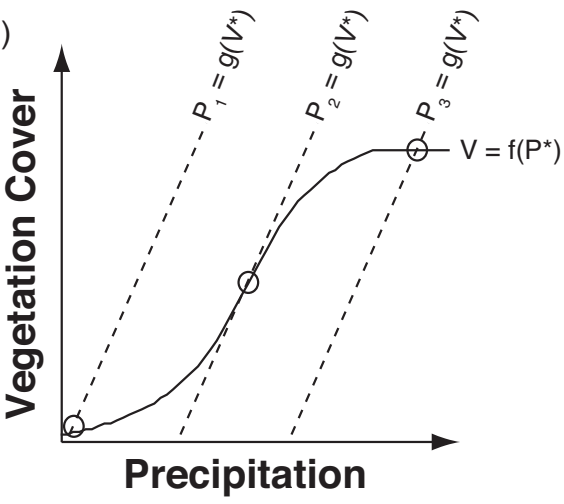

Fig. 1. Conceptual diagram to illustrate the effect of vegetation parameterizations on the emergence of multiple steady states. Diagram (a) shows the case in which vegetation is parameterized by a few discrete vegetation classes while (b) uses a continuous representation of vegetation. The solid lines represent vegetation cover $V=f\left(P^{*}\right)$ as a function of a fixed, given value of precipitation $P^{*}$. The dashed lines represent precipitation $P=g\left(V^{*}\right)$ as a function of a fixed, given value of vegetation cover $V^{*}$ for three different precipitation regimes

boreal forest results in climatic conditions more suitable for vegetation growth, which in turn favors the presence of the forest. The possibility of multiple steady states at the boreal forest-tundra boundary was investigated for present-day conditions by Levis et al. (1999) and Brovkin et al. (2003), although both studies found only one possible steady state in the high latitude regions.

2. In regions where water limits productivity (primarily in the seasonal tropics), the presence of vegetation results in enhanced evapotranspiration and continental moisture cycling, affecting the larger-scale circulation (e.g. Charney, 1975). This also results in a positive feedback, where the presence of vegetation results in climatic conditions more suitable for vegetation growth through reducing the effect of water limitation, thus favoring the presence of vegetation. Multiple steady states in this transition region of West Africa were investigated and demonstrated by Claussen (1994), Claussen (1997), Claussen (1998), Brovkin et al. (1998), Zeng and Neelin (2000), Wang and Eltahir (2000a), Wang and Eltahir (2000b), Wang (2004), and Zeng et al. (2004).

The emergence of multiple steady states associated with vegetation feedbacks can be understood conceptually as follows (Brovkin et al., 1998; Wang, 2004). Let us characterize the vegetation state by the fractional cover of vegetation, $V$, which is closely tied to surface albedo and other aspects of land surface functioning. The presence of forest in the boreal region allows to mask the effect of snow, and thereby impacts the albedo of the surface. In semiarid regions, the presence of vegetation results in a lower surface albedo. Consequently, in both cases, $V$ can be directly seen as a proxy for the ability of the vegetated surface to maintain a low albedo, and is therefore linked to a fundamental parameter affecting the climatic state.

Vegetation cover depends on vegetation productivity. In semiarid regions productivity is limited primarily by the availability of water, and is therefore a function of precipitation $P$. For a fixed, given amount of precipitation $P^{*}$ (with the asterisk indicating that the amount of precipitation is treated as an independent variable in this case) we can plot a line $V=f\left(P^{*}\right)$ as shown in Fig. 1 by the solid lines.

A common way to simulate vegetation-related properties is to use a biome classification or biome-averaged vegetation properties. In such a classification, the vegetated state may be characterized by a few possible values for $V$, each representative of the mean value for the respective biome. Consequently, the functional relationship $V=f\left(P^{*}\right)$ is essentially a step function as shown in Fig. 1a. A continuous parameterization for $V=f\left(P^{*}\right)$, which would account for a mixture of vegetation types and its diversity within a region, would likely lead to a smooth line (see Fig. 1b).

On the other hand, $V$ affects land surface functioning, through land surface parameters such as surface albedo, aerodynamic roughness, and rooting zone depth. These attributes affect the overlying atmosphere, and thereby the rate of precipitation. For a fixed given value of $V^{*}$, one can plot the relationship $P=g\left(V^{*}\right)$ for different precipitation regimes, indicated by $P_{1}, P_{2}$, and $P_{3}$ in Fig. 1 (dashed lines). The sensitivity of precipitation $P$ to different values of $V^{*}$ is represented by the steepness of the curve, with a vertical line representing the case of no dependence of $P$ on $V^{*}$.

Possible solutions to the coupled water- and carbon balances - characterized by $P$ and $V$ respectively - are given by the intersection of the lines $V=f(P)$ and $P=g(V)$ in Fig. 1 . If this were not the case, the water- and carbon balances would not be in a steady state and would evolve towards one. Imagine a point $\left(V^{\prime}, P^{\prime}\right)$ for which this is not the case. If $V^{\prime}>f\left(P^{\prime}\right)$, then this would imply that either the biomass $V^{\prime}$ cannot be sustained with the given amount of precipitation $P^{\prime}$, or that a given vegetation cover $V^{\prime}$ would result in a precipitation rate that is greater than $P^{\prime}$ (because $g\left(V^{\prime}\right)>P^{\prime}$ ), or 
a combination of both. Consequently, the water- and carbon balances would evolve towards a point $\left(P^{\prime \prime}, V^{\prime \prime}\right)$ at which $V^{\prime \prime}=f\left(P^{\prime \prime}\right)$ and $P^{\prime \prime}=g\left(V^{\prime \prime}\right)$.

As we can see from Fig. 1, the representation of vegetation by a discrete number of "biome" classes leads to multiple steady states for the precipitation regime indicated by $P_{2}$, even though a smooth, continuous representation of vegetation would only lead to one intersection of the curves, and therefore to only one possible steady state. From this conceptual example we can conclude that the way that vegetation is treated in a climate model plays a critical role on whether multiple steady states can emerge from the model dynamics. This seemingly artificial example is still relevant to investigate since some previous studies effectively used such discrete vegetation schemes. For instance, the study by Claussen (1994) coupled the ECHAM climate model to the BIOME model Prentice et al. (1992), which maps surface climate into 17 biome types, thereby effectively resulting in a discrete vegetation classification. Note, however, that depending on the sensitivity of $P$ to $V^{*}$ (i.e. the value of $d P / d V^{*}$ or the slope of the dashed lines), multiple intersections are possible, hence also multiple steady states. Here, however, we focus on the effect of a discrete classification of vegetation only, for which multiple steady states can emerge simply due to the way of how vegetation is represented in the model.

Here, we use a coupled dynamic vegetation-atmosphere model of intermediate complexity to investigate the role of vegetation classes in the emergence of multiple steady states. To do so, we convert a continuous parameterization that maps vegetation biomass into land surface parameters into one of a few discrete classes. A brief description of our model and how we convert a continuous representation of vegetation into discrete classes is described in the next section. In the results section we present the climatic differences associated with different initial conditions, how these climatic differences depend on the prescribed number of vegetation classes and discuss how these relate to the two biogeophysical feedback loops discussed above. We close with a brief summary and conclusion.

\section{Methods}

\subsection{The Planet Simulator}

We use the Planet Simulator Earth system model of intermediate complexity (Lunkeit et al., 2004; Fraedrich et al., 2005a,b). The Planet Simulator consists of a low resolution atmospheric general circulation model, a mixed-layer ocean model, a thermodynamic sea-ice model, and a land surface model. The atmospheric model consists of a dynamical core and a physical parameterization package of intermediate complexity for unresolved processes. We use the atmospheric model in its $\mathrm{T} 21$ resolution (corresponding to approx. $5.625^{\circ} \cdot 5.625^{\circ}$ longitude/latitude resolution) and 5 vertical layers. Over land, a 5 layer heat diffusion model simulates soil temperature, and a "bucket" model simulates soil hydrology. A dynamic global vegetation model (SimBA) simulates the effect of climate on vegetation-affected land surface parameters, such as surface albedo, surface roughness, and the total soil water holding capacity.

In this study, we use the Planet Simulator in a setup with prescribed sea surface temperatures. We chose this setup because the effect of oceanic feedbacks on multiple steady states in vegetation is not the main aspect of this study. The model leads to a reasonable simulated climate and vegetation distribution for present-day forcings (see Fig. 2), although some regional deficiencies exist (e.g. dryness of West Africa and central Amazonia). However, for the more conceptual nature of our study, we take this control simulation as being sufficiently suitable for the purpose here. More importantly the dominant climatic effects and feedback mechanisms related to differences in vegetation are consistent with those of previous studies (Fraedrich et al., 2005b; Kleidon, 2006a,b).

\subsection{Discrete versus continuous vegetation classes}

The SimBA dynamic vegetation model simulates regional vegetation biomass $C_{\text {veg }}$ from the balance of carbon exchange fluxes. Carbon uptake by gross primary productivity GPP depends on incoming photosynthetically active solar radiation, surface temperature, and soil moisture status. Autotrophic respiration is assumed to be $50 \%$ of GPP, which is commonly observed and can be understood by optimum nitrogen allocation in canopies (Dewar, 1996). Litter production is expressed as $C_{\text {veg }} / \tau_{\text {veg }}$ with $\tau_{\text {veg }}$ being a fixed turnover time of 10 years. As a consequence, mean vegetation biomass is proportional to mean productivity in our model.

Climatically-relevant land surface parameters are derived mainly from the fractional cover of vegetation $f_{\text {veg }}$. The vegetation cover is derived from biomass in the standard version of the model by:

$f_{\mathrm{veg}}=\frac{1}{c_{c}} \cdot \arctan \left(\frac{C_{\mathrm{veg}}-c_{a}}{c_{b}}\right)+c_{d}$

In Eq. (1), $c_{a}, c_{b}, c_{c}$, and $c_{d}$ are empirical parameters which were derived by matching the resulting distribution of woody vegetation with the observed vegetation distribution. Vegetation cover is then used to derive, for instance, the soil moisture holding capacity of the rooting zone $W_{\max }$ by $W_{\text {max }}=f_{\text {veg }} \cdot W_{\text {max } \text { veg }}+\left(1-f_{\text {veg }}\right) \cdot W_{\text {max, noveg, with }} W_{\text {max }, \text { veg }}$ being the value for a surface that is completely covered by vegetation, and $W_{\text {max, noveg }}$ being the respective value for a surface in the absence of woody vegetation. Other land surface parameters, specifically leaf area index and leaf cover, surface albedo and roughness, are derived in an analogous way. For details of the vegetation model, see Lunkeit et al. (2004) and Kleidon (2006a). 

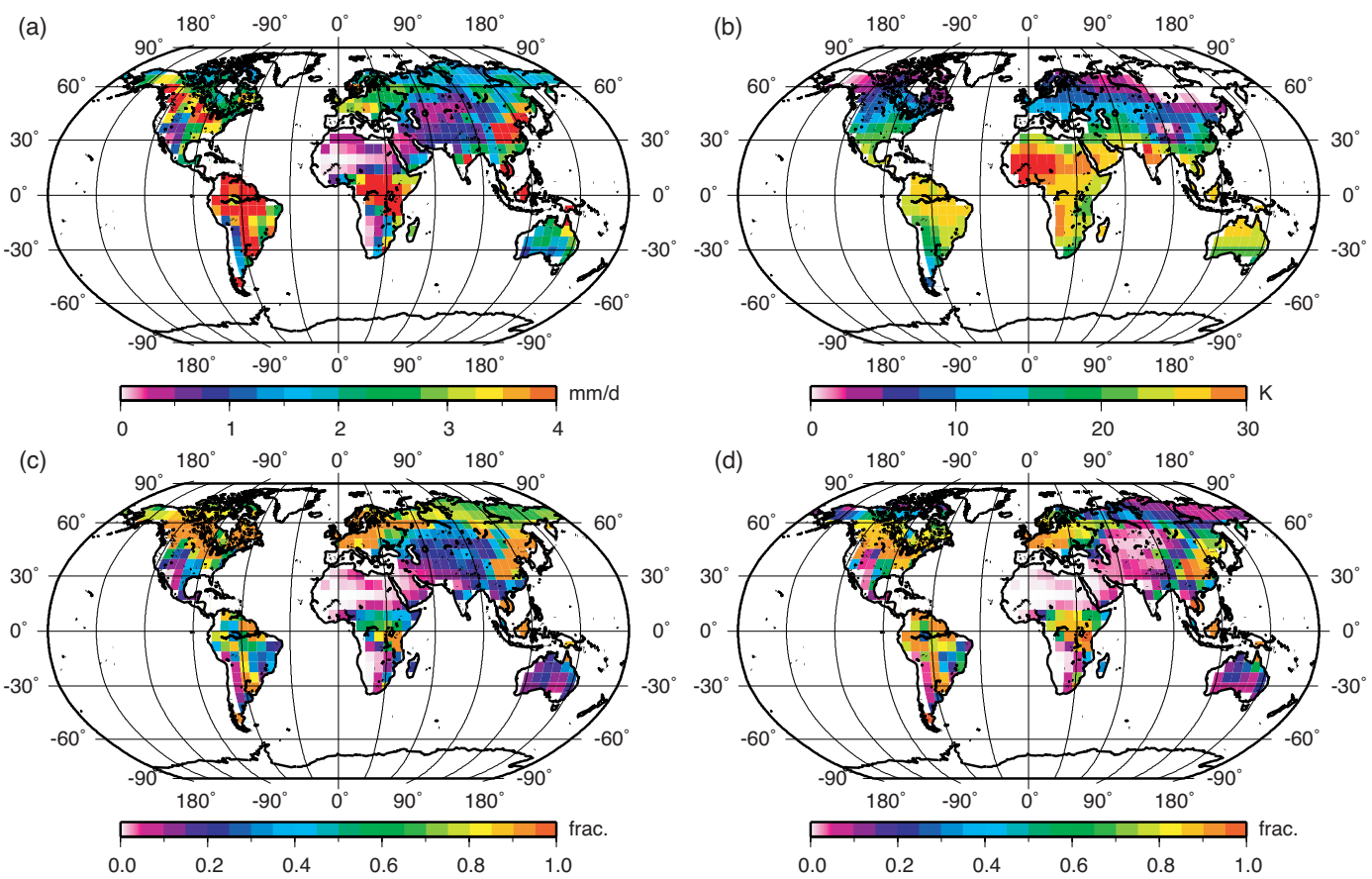

Fig. 2. Simulated climate of the control setup of the Planet Simulator in T21L5 setup. The graphs show annual means of (a) precipitation; (b) near surface air temperature; (c) leaf cover; and (d) vegetation cover.

In order to introduce discrete vegetation classes, we rewrite $f_{\text {veg }}$ in discrete form (which we refer to as $f_{\text {veg, } d}$ ):

$f_{\mathrm{veg}, d}=\frac{\inf \left(f_{\mathrm{veg}} \cdot n\right)}{n-1}$

where $\inf (x)$ denotes the truncation of the expression $x$ to its integer value, and $n$ is the specified number of vegetation classes. What Eq. (2) results in is a mapping of biomass to a finite number of $n$ fractional cover values that then result in a discrete set of land surface parameters for certain ranges of vegetation biomass.

\subsection{Simulation setup}

We conduct a series of sensitivity simulations which differ by (a) the initialization of the vegetation state $\left(C_{\mathrm{veg}}=0\right.$ in a "bare ground" initialization and $C_{\mathrm{veg}}=5 \mathrm{~kg} \mathrm{C} \mathrm{m}^{-2}$ in a "fully vegetated" initialization) and (b) the number of discrete vegetation classes ( $n$ in Eq. 2). We use values of $n=2,3,4$, $5,6,7,8$, and 10 . The continuous vegetation formulation of Eq. (1), which we will refer to as $n=\infty$, is taken as our "Control" simulation.

Each sensitivity simulation is run for 40 years. For the first 30 years the time step of the vegetation model is increased in order to accelerate the transition to a steady-state. In this acceleration scheme, we use the time step of the atmospheric model for the first 2 years to allow for the physical climatic variables to come close to a steady-state. For year 3 to 8 , we use a factor of 20 to accelerate the carbon pool dynam- ics, so that the simulated time of 5 years corresponds to 100 years of vegetation dynamics. In the next 5 year intervals, we subsequently decrease the acceleration factor to 10,5 , and 2 . During the remaining time, the vegetation model is run at the atmospheric time step. With this setup, we achieve an "effective" simulation period for vegetation dynamics of more than 200 years. With additional sensitivity simulations we confirmed that this acceleration scheme does not affect the outcome of the final steady state (not shown). The motivation for the subsequently decreased time step is that the acceleration of the carbon dynamics also leads to an increase in the variability in the carbon pools. With the decreased acceleration factor in later stages of the simulation, this variability is reduced.

We use averages taken over the last 10 years of the simulation period to evaluate the differences in the steady states.

\section{Results and discussion}

The sensitivity of the land climate to vegetation classes is summarized in Fig. 3. These averages show clear differences to the "Control" simulation in simulated vegetation productivity, radiative fluxes, water fluxes, fractional vegetation cover, and near surface air temperature with increasing values of vegetation classes $n$. The simulations with discrete vegetation classes generally show lower gross primary productivity of $0.8-1.6 \mathrm{~g} \mathrm{C} \mathrm{m}^{-2} \mathrm{~d}^{-1}$ compared to the average value of $1.8 \mathrm{~g} \mathrm{C} \mathrm{m}^{-2} \mathrm{~d}^{-1}$ in the "Control". In terms of the 

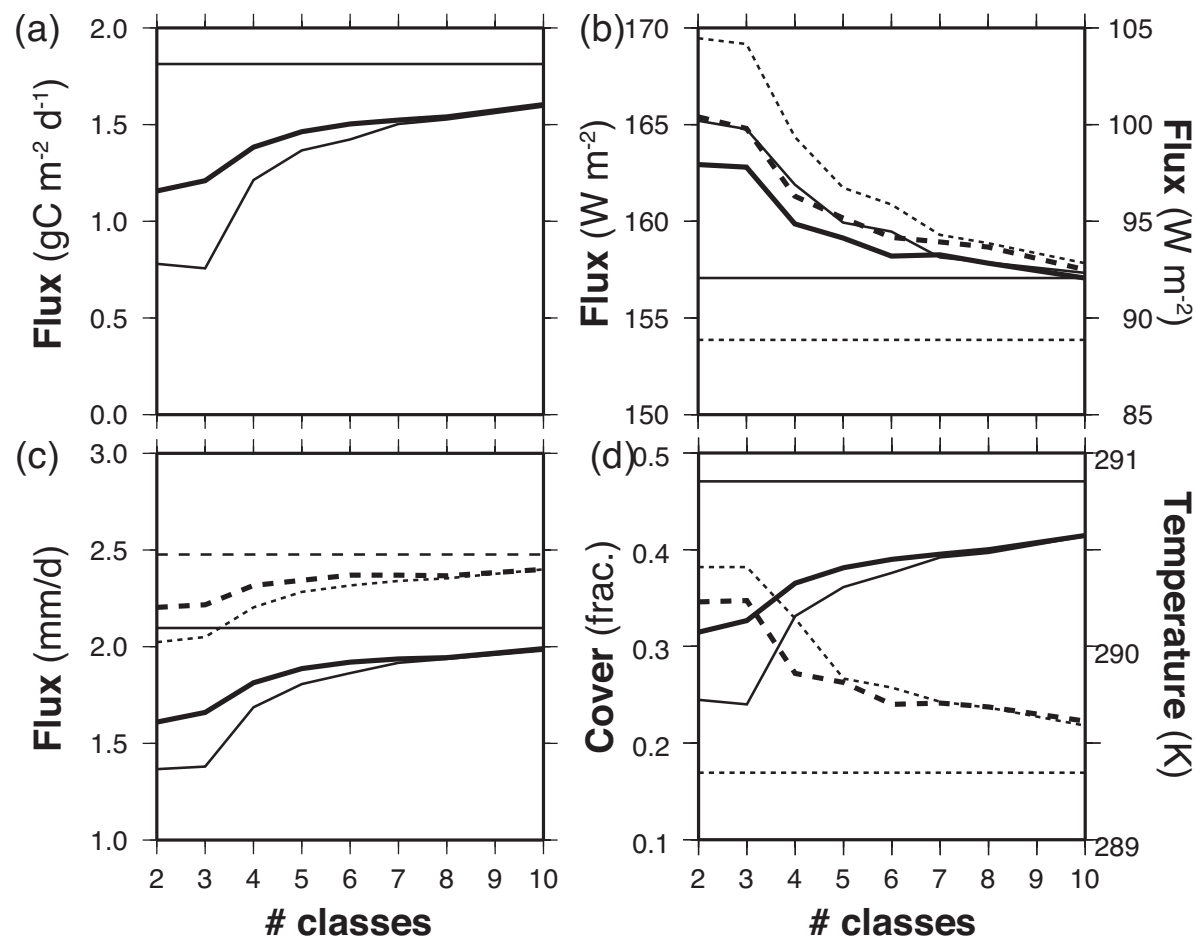

Fig. 3. Sensitivity of annual mean land averages of (a) gross primary productivity, (b) net solar (solid line, left scale) and terrestrial (dashed line, right scale, positive = radiative cooling) radiation at the surface, $(\mathbf{c})$ evapotranspiration (solid) and precipitation (dashed), and (d) vegetation cover (solid) and near surface air temperature (dashed) to the number of vegetation classes $n$. The thick (thin) lines show the sensitivity for the initialization of fully (bare) vegetation cover respectively. The horizontal lines indicate the respective values of the "Control" simulation.

surface energy balance, the net flux of solar and terrestrial radiation is higher by up to $8 \mathrm{~W} \mathrm{~m}^{-2}$ and $16 \mathrm{~W} \mathrm{~m}^{-2}$, respectively. Precipitation and evapotranspiration are lower by as much as $0.8 \mathrm{~mm} \mathrm{~d}^{-1}$ and $0.5 \mathrm{~mm} \mathrm{~d}^{-1}$, respectively. Global land temperatures show a minor but consistent trend of being warmer by up to $\approx 1 \mathrm{~K}$. The climatic differences due to initial conditions are generally less than those between the discrete vegetation classification and the "Control".

Two general trends can be seen in Fig. 3: first, the difference due to the initial conditions diminishes with increasing values of $n$, and disappears for $n=8$ or more classes. The other trend is that the steady state climates asymptotically approximate the "Control" climate. However, even after the multiple steady states converge into a single steady state at $n=8$, the climate still differs from the "Control", resulting in climatic conditions that result in lower values of gross primary productivity. However, the results do not show that more steady-state solutions exist as $n$ increases as hypothesized in Fig. 1b. This would require additional model simulations with a wider range of initial conditions.

These climatic differences are consistent and can be explained as follows: As productivity increases with $n$, so does evapotranspiration and leaf cover. As the land surface is cooled more effectively by evapotranspiration, the net emis- sion of terrestrial radiation decreases. The increase of evapotranspiration in turn leads to (a) lower surface temperatures due to enhanced latent cooling, (b) enhanced continental precipitation, and (c) increased cloud cover (not shown). The increase in cloud cover results in lower absorption of solar radiation, even though surface albedo decreases with increased leaf cover.

Figure 4 shows where and by how much the steady state vegetation biomass $C_{\text {veg }}$ differs due to different initial conditions and different number of vegetation classes. Note that differences in $C_{\text {veg }}$ represent differences in vegetation productivity, because the model uses a fixed residence time to simulate biomass (see Sect. 2). In the extreme case of $n=2$, vegetation biomass is substantially reduced compared to the "Control" across all regions. This reduction is still present in the case of $n=8$, but less pronounced. The differences in biomass due to initial conditions for $n=2$ can be found in tropical South America, China, western Europe, and North America. The differences in biomass for $n=8$ are comparatively minor.

These differences in steady state biomass result from differences in the dominant factors that limit productivity. The dominant limitation in the high latitudes is the length of the growing season. The difference in vegetation states affect 

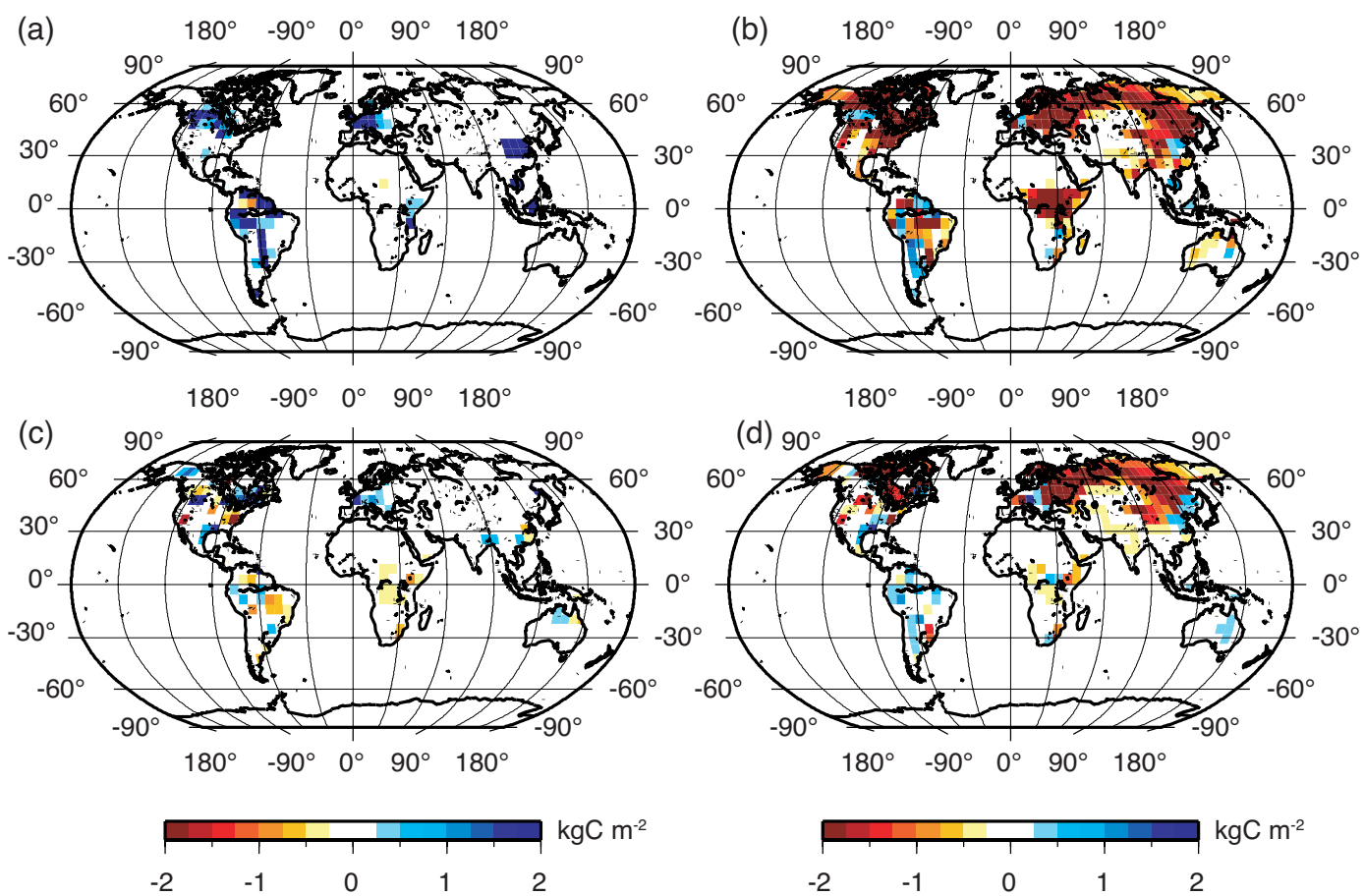

Fig. 4. Differences in vegetation biomass for (a) "full vegetation" - "bare ground" initializations for $n=2$ (b) same as (a), but for "full vegetation" initialization - "Control", (c) and (d) are the same as (a) and (b), but for $n=8$.
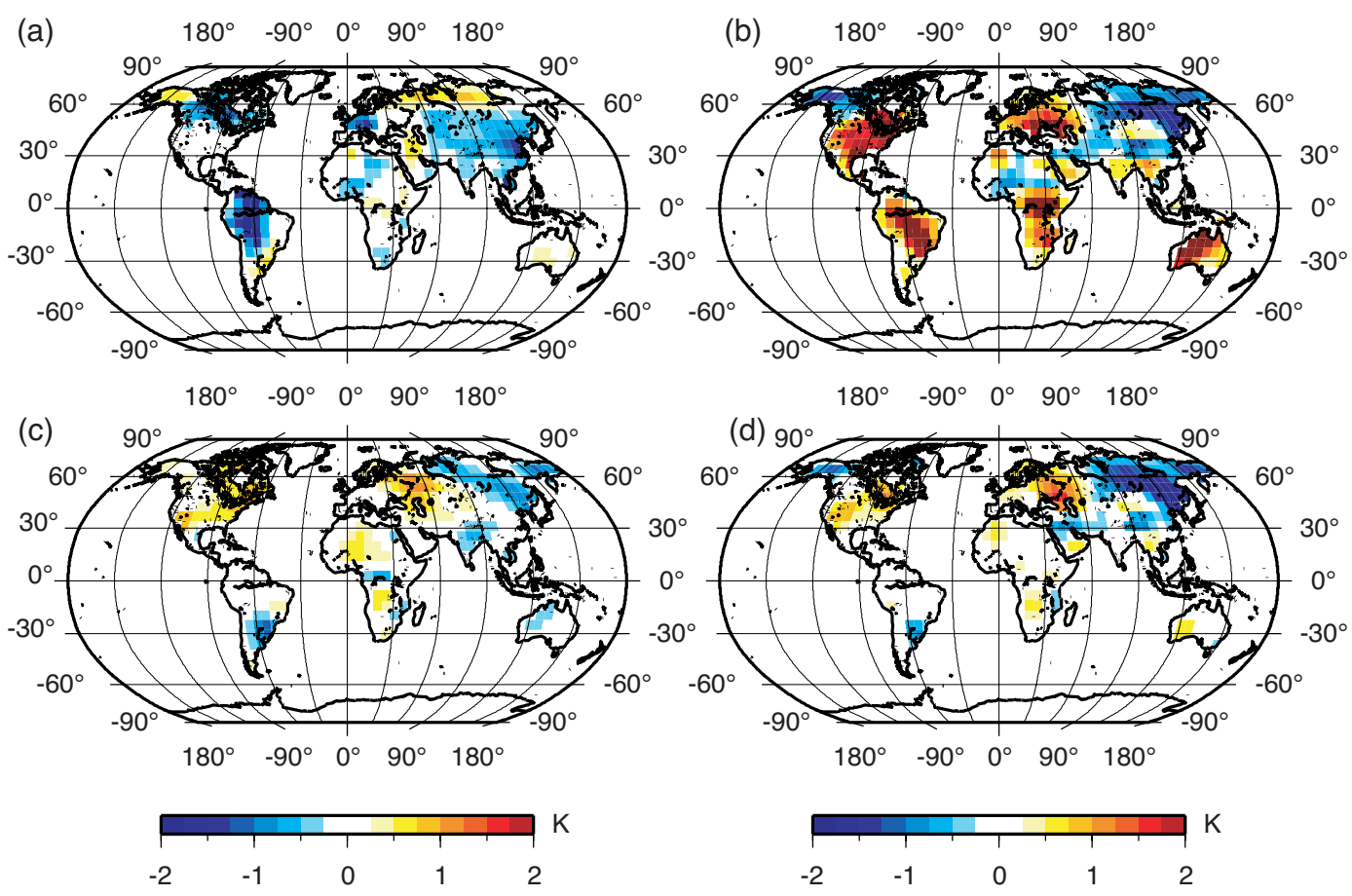

Fig. 5. Same as Fig. 4, but for Northern Hemisphere springtime (March-May) near surface air temperature.

primarily the amount of absorbed solar radiation in the presence of snow, thereby affecting springtime temperatures. The differences in Northern Hemisphere springtime temperatures
$T_{\text {mam }}$ (March-May) are shown in Fig. 5. These results show that $T_{\text {mam }}$ is relatively insensitive to initial conditions for $n=2$ and $n=8$. Compared to the "Control", both cases show 

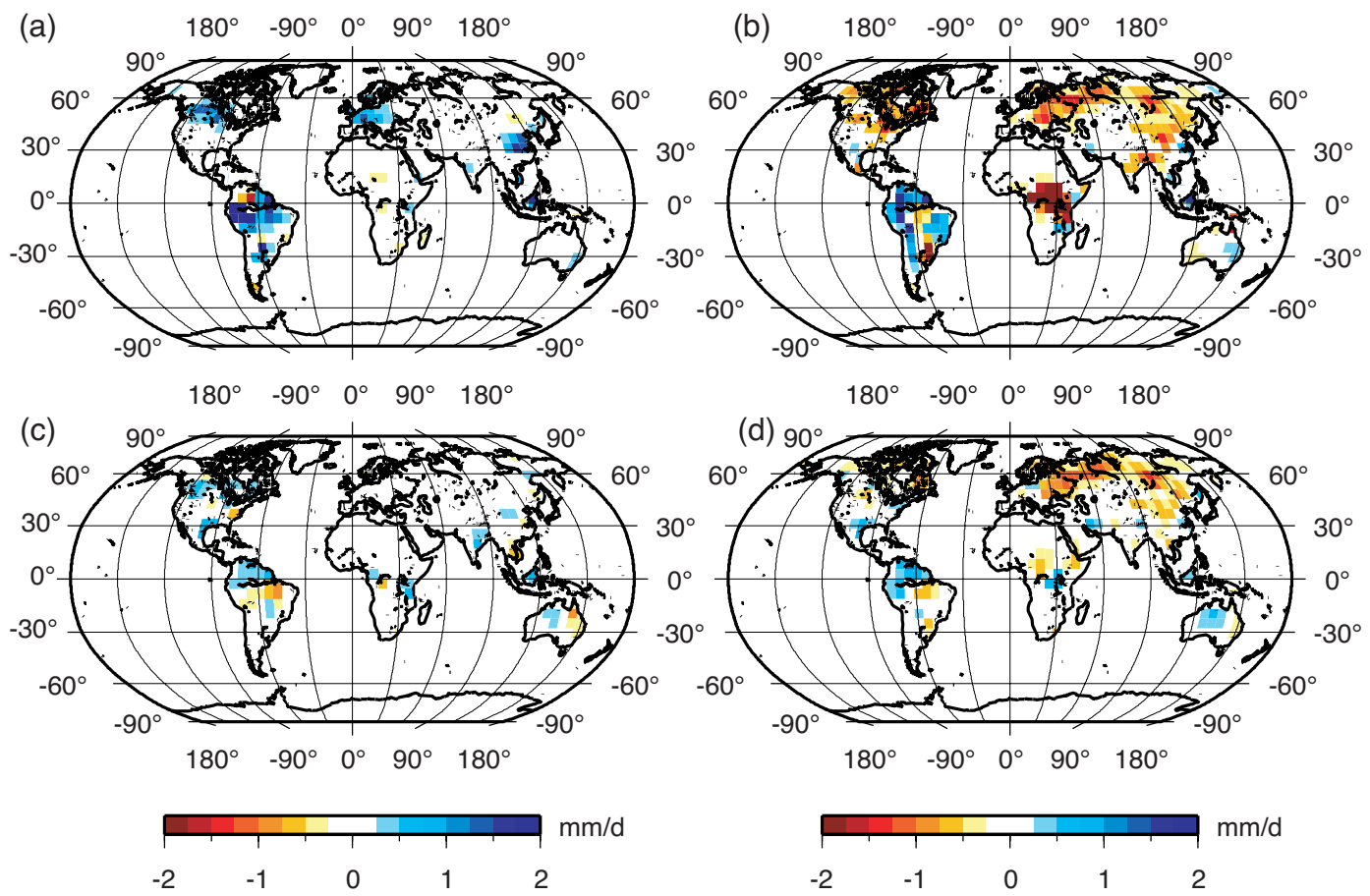

Fig. 6. Same as Fig. 4, but for annual mean precipitation.

large regions in the high latitudes for which $T_{\text {mam }}$ are colder by $2 \mathrm{~K}$ and more. These differences correspond well to the differences in biomass shown in Fig. 4. This leads us to conclude that the vegetation and climate differences in these regions are primarily caused by the effect of vegetation on snow albedo and growing season length.

The differences in biomass due to initial conditions for $n=2$ can be attributed primarily to differences in water availability, as shown in the differences in annual mean precipitation (Fig. 6). The locations of higher biomass in Fig. 4a correspond well with those of higher precipitation in Fig. 6a. Similarly, differences in biomass compared to the "Control" can generally be attributed to differences in precipitation for tropical regions (and to some extent for temperate regions), and thus linked with the dominant control of water availability on productivity.

\section{Conclusions}

This paper shows that multiple steady states in the coupled vegetation-atmosphere system can emerge as a result of a discrete representation of vegetation form and function in an Earth system model of intermediate complexity, as hypothesized in Fig. 1. The two major positive vegetation feedbacks that result in the emergence of these steady states are related to the temperature limitation on productivity in high latitudes and the water limitation in the tropics and subtropics. This finding is consistent with previous studies.
These results have two important implications for representing vegetation dynamics in Earth system models. First, multiple steady states in the vegetation-atmosphere system may simply be model artefacts that disappear if the full complexity and heterogeneity in vegetation form and functioning and its ability to adapt to the prevailing climatic conditions is fully represented in the model. While the model setup in this study only allowed for one steady-state to emerge as a result of a continuous vegetation parameterization, the study by Kleidon (2006b) demonstrates that given reasonable ranges of vegetation parameters, a whole, continuous range of steady states are possible to emerge in which vegetation can maximize its productivity. This may point out that the concept of multiple steady states may be limited in its applicability to adaptive vegetation, but this would require further investigations. Second, a discrete representation of vegetation seems to result in a general underestimation of terrestrial productivity. To identify the reasons why this is so would also require further investigations. Nevertheless, both of these implications are important to keep in mind when we want to understand the overall response of the terrestrial biosphere to past and future climatic changes.

Acknowledgements. We thank three anonymous reviewers and K. Caldeira for their constructive comments. This research was initiated as part of the Undergraduate Research Assistance Program of the University of Maryland. Funding was provided to A. Kleidon by the NSF climate dynamics program through grant ATM 0513506. K. Fraedrich acknowledges support by DFG-Project FR 450/11-1. 
Edited by: A. Neftel

\section{References}

Bonan, G. B., Pollard, D., and Thompson, S. L.: Effects of boreal forest vegetation on global climate, Nature, 359, 716-718, 1992.

Brovkin, V., Claussen, M., Petoukhov, V., and Ganopolski, A.: On the stability of the atmosphere-vegetation system in the Sahara/Sahel region, J. Geophys. Res., 103(D24), 31 613-31 624, 1998.

Brovkin, V., Levis, S., Loutre, M.-F., Crucifix, M., Claussen, M., Ganopolski, A., Kubatzki, C., and Petoukhov, V.: Stability analysis of the climate-vegetation system in the northern high latitudes, Clim. Ch., 57, 119-138, 2003.

Budyko, M. I.: The Effect of Solar Radiation Variations on the Climate of the Earth, Tellus, 21, 611-619, 1969.

Charney, J. G.: Dynamics of deserts and drought in the Sahel, Q. J. Roy. Meteor. Soc., 101, 193-202, 1975.

Claussen, M.: On coupling global biome models with climate models, Clim. Res., 4, 203-221, 1994.

Claussen, M.: Modeling bio-geophysical feedback in the African and Indian monsoon region, Clim. Dynam., 13, 247-257, 1997.

Claussen, M.: On multiple solutions of the atmosphere-vegetation system in present-day climate, Global Chem. Biol., 4, 549-559, 1998.

Dewar, R. C.: The correlation between plant growth and intercepted radiation: an interpretation in terms of optimal plant nitrogen content, Ann. Bot., 78, 125-136, 1996.

Fraedrich, K.: Catastrophes and resilience of a zero-dimensional climate system with ice-albedo and greenhouse feedback, Q. J. Roy. Meteor. Soc., 105, 147-167, 1979.

Fraedrich, K., Jansen, H., Kirk, E., Luksch, U., and Lunkeit, F.: The Planet Simulator: Towards a user friendly model, Z. Meteorol., 14, 299-304, 2005a.

Fraedrich, K., Jansen, H., Kirk, E., and Lunkeit, F.: The Planet Simulator: Green planet and desert world, Z. Meteorol., 14, 305314, 2005b.

Harvey, L. D. D.: On the role of high latitude ice, snow, and vegetation feedbacks in the climatic response to external forcing changes, Clim. Change, 13, 191-224, 1988.

Kleidon, A.: The climate sensitivity to human appropriation of vegetation productivity and its thermodynamic characterization, Global Planet. Ch., 54, 109-127, 2006a.
Kleidon, A.: Quantifying the biologically possible range of steadystate soil and surface climates with climate model simulations, Biologia, Bratislava, 61/Suppl. 19, S234-239, 2006b.

Kleidon, A. and Fraedrich, K.: Biotic Entropy Production and Global Atmosphere-Biosphere Interactions, in: NonEquilibrium Thermodynamics and the Production of Entropy: Life, Earth, and Beyond, edited by: Kleidon, A. and Lorenz, R. D., pp. 173-190, Springer Verlag, Heidelberg, Germany, 2005.

Levis, S., Foley, J. A., Brovkin, V., and Pollard, D.: On the stability of the high-latitude climate-vegetation system in a coupled atmosphere-biosphere model, Global Ecol. Biogeog., 8, 489500, 1999.

Lunkeit, F., Fraedrich, K., Jansen, H., Kirk, E., Kleidon, A., and Luksch, U.: Planet Simulator Reference Manual, available at http://puma.dkrz.de/plasim, 2004.

Otterman, J., Chou, M. D., and Arking, A.: Effects of nontropical forest cover on climate, J. Clim. Appl. Meteorol., 23, 762-767, 1984.

Prentice, I. C., Cramer, W., Harrison, S. P., Leemans, R., Monserud, R. A., and Solomon, A. M.: A global biome model based on plant physiology and dominance, soil properties and climate, J. Biogeography, 19, 117-134, 1992.

Otterman, J., Chou, M. D., and Arking, A.: Effects of nontropical forest cover on climate, J. Clim. Appl. Meteorol., 23, 762-767, 1984.

Sellers, W. D.: A Global Climatic Model Based on the Energy Balance of the Earth-Atmosphere System, J. Appl. Meteorol., 8, 392-400, 1969.

Wang, G.: A conceptual modeling study on biosphere-atmosphere interactions and its implications for physically based climate modeling, J. Climate, 17, 2572-2583, 2004.

Wang, G. and Eltahir, E. A. B.: Biosphere-atmosphere interactions over West Africa. 2. Multiple climate equilibria, Q. J. Roy. Meteor. Soc., 126, 1261-1280, 2000a.

Wang, G. and Eltahir, E. A. B.: Ecosystem dynamics and the Sahel drought, Geophys. Res. Lett., 27, 795-798, 2000b.

Zeng, N. and Neelin, J. D.: The role of vegetation-climate interaction and interannual variability in shaping the African savanna, J. Climate, 13, 2665-2670, 2000.

Zeng, X., Shen, S. S. P., Zeng, X., and Dickinson, R. E.: Multiple equilibrium states and the abrupt transitions in a dynamical system of soil water interacting with vegetation, Geophys. Res. Lett., 31, L05 501, doi:10.1029/2003GL018910, 2004. 